Rechtsmedizin 2021 · 31:494-500 https://doi.org/10.1007/s00194-021-00461-0 Angenommen: 28. Dezember 2020 Online publiziert: 16. Februar 2021 (c) Der/die Autor(en) 2021

\author{
C. Nentwig' $\cdot$ A. Schütz ${ }^{1}$ S. Steinhoff ${ }^{2}$ J. Adamec ${ }^{3} \cdot$ S. N. Kunz ${ }^{1,4}$ \\ 'Universität Ulm, Ulm, Deutschland \\ ${ }^{2}$ Abteilung für Radiologie, Bundeswehrkrankenhaus, Ulm, Deutschland \\ ${ }^{3}$ Institut für Rechtsmedizin, Ludwig-Maximilians-Universität, München, Deutschland \\ ${ }^{4}$ Institut für Rechtsmedizin des Universitätsklinikums UIm, Ulm, Deutschland
}

Die rechtsmedizinische Begutachtung von Verletzungen im Kopfbereich durch Schläge mit Bierflaschen aus Glas, nachfolgend als Bierflaschen abgekürzt, ist oftmals die Folge körperlicher Auseinandersetzungen in Schankgaststätten. Im Rahmen der juristischen Aufarbeitung eines entsprechenden Sachverhaltes stellen sich die Fragen nach der tatsächlich erfolgten Tathandlung und der akuten sowie potenziellen Lebensgefahr einer getroffenen Person. Ziel forensisch-biomechanischer Begutachtung ist es, die entstandenen Verletzungen hinsichtlich ihrer Schwere zu beurteilen und einen Entstehungsmechanismus zu rekonstruieren. In Abhängigkeit des verwendeten Tatwerkzeuges, der Schlagtechnik, der eingesetzten Kraft und der Kontaktfläche mit einer getroffenen Körperregion gilt es, die Verletzungsgefahr durch diese spezielle Form der Gewalteinwirkung zu beurteilen. In der rechtsmedizinischen Literatur gibt es bis dato keine ausreichende, experimentell validierte Datenmenge, mithilfe derer entsprechende Fragestellungen, bezogen auf gebrauchte, leere 0,5-1-Bierflaschen, forensisch-biomechanisch beantwortet werden können. Grundsätzlich ist es schwierig, menschliches Originalgewebe zu Testzwecken $\mathrm{zu}$ erhalten. Deshalb ist es von besonderer Wichtigkeit, zielführende Versuche an vergleichbaren Substanzen wie Acryl oder Schweineschwarte auf Kopfmodellen durchzuführen.
Die vorliegende Arbeit setzt sich mit den forensisch-biomechanischen Aspekten von Schlägen mit gebrauchten, leeren 0,5-1-Bierflaschen gegen den menschlichen Schädel auseinander. Mehrere experimentelle Untersuchungen liefern im Folgenden eine Interpretationsgrundlage, um den Vorgang eines Bierflaschenschlags gegen den Kopf rekonstruieren und das Verletzungspotenzial in Abhängigkeit der übertragenen Energie und des Bruchverhaltens beurteilen zu können.

\section{Material und Methoden}

30 gebrauchte, leere 0,5-1-Bierflaschen aus Glas der Augustiner Brauerei München mit einem Gewicht von ca. $345 \mathrm{~g}$ wurden als Schlagwerkzeug verwen$\operatorname{det}(\bullet$ Abb. 1). Die Bierflaschen wurden am Flaschenhals gehalten und manuell in einer Abwärtsbewegung gegen das Schädelsimulanz, einen Alukern eines Dummy-Kopfes Hybrid III (Humanetics ATD manufacturing, Huron, $\mathrm{OH}$, USA), geschlagen. Als Kopfhautsurrogat wurde Acryl mit einer Dicke von $3 \mathrm{~mm}$ in den Versuchen 1-20 auf den Kopf gezogen. In den Versuchen 21-30 wurden zum Vergleich $5 \mathrm{~mm}$ dicke Schweineschwarten verwendet. Der präparierte Aluminiumschädel wurde auf der Mehrkomponenten-Kraftmessplatte Typ 9286B der Fa. Kistler Instrumente AG (Kistler Instrumente $\mathrm{GmbH}$, Sindelfingen, Deutschland) befestigt (- Abb. 2). Mit der BioWare ${ }^{\circledR}$-Software (Kistler Instru- mente GmbH, Sindelfingen, Deutschland) wurde die Bodenreaktionskraft bis $\mathrm{zu}$ einem Maximalwert von $12 \mathrm{kN}$ aufgezeichnet. Fotos wurden mit der Digitalkamera Canon EOS 250D (Canon $\mathrm{GmbH}$ Deutschland, Krefeld) angefertigt.

5 weibliche und 2 männliche Probanden führten die Schläge abwechselnd in einer Vertikalbewegung aus, wobei die Flasche jeweils am Hals gehalten wurde. Die Kontaktfläche war die zylindrische Seitenfläche der Bierflaschen jeweils mit der Scheitelregion des Dummy-Kopfes. Blieb die Flasche intakt, wurde der Versuch mit (subjektiv) erhöhter Intensität und gleichbleibender Bewegungsführung erneut bis zum Bruch der Flasche ausgeführt. Ziel war es, die maximal mögliche Kraftübertragung auf den Schädel herauszufinden. Zusätzlich wurden Verletzungen an den Kopfhautsurrogaten sowie die Bruchmuster der Bierflaschen analysiert.

\section{Ergebnisse}

Die Schläge mit leeren Bierflaschen aus Glas auf den mit Acryl überzogenen Schädel und auf den mit Schweineschwarte präparierten Schädel lieferten vergleichbare Ergebnisse. Im Fall des Acrylsurrogats zeigte sich nach 20 Versuchen (•Tab. 1) eine durchschnittliche Bruchkraft von 532 N (318-921 N). Nach 10 Schlägen auf die Schweineschwarte konnte eine durchschnittliche Bruch- 


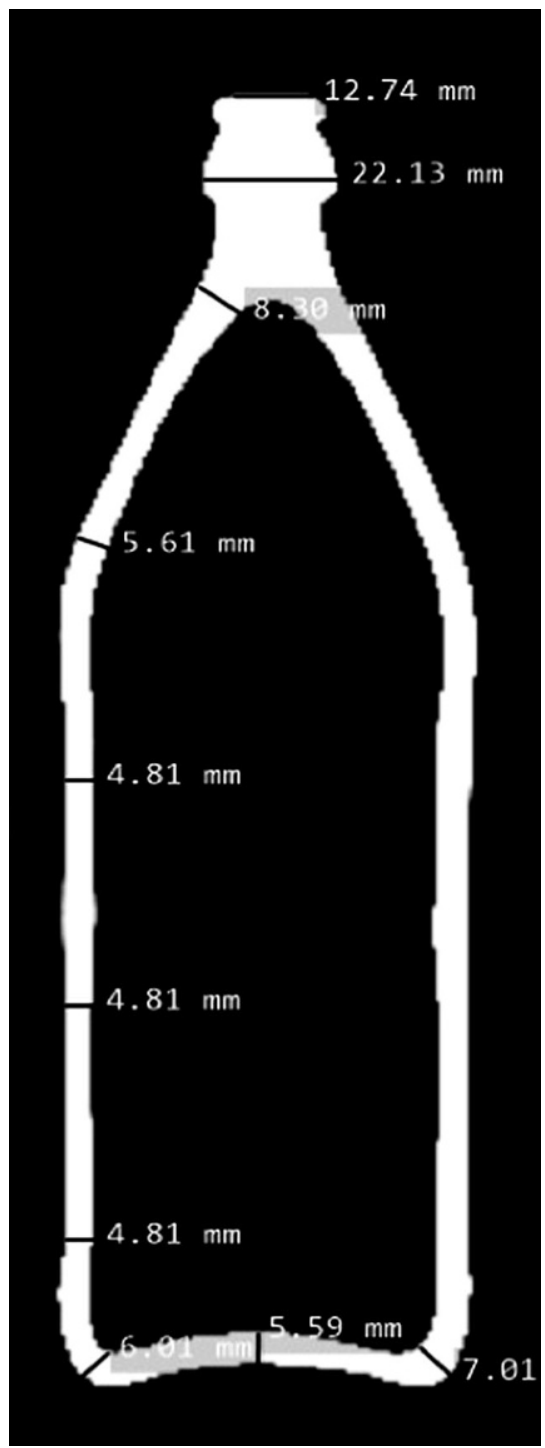

Abb. $1 \Delta$ Computertomographische Untersuchung einer 0,5-I-Bierglasflasche der Augustiner Brauerei München

kraft von $750 \mathrm{~N}(394-951 \mathrm{~N})$ ermittelt werden (•Tab. 2). Es stellte sich heraus, dass ein Zerbrechen der Flasche in der Regel mit einer Reduktion der auf den Kopf übertragenen Kraft einhergeht. Die höchste Transferkraft wurde bei intakten Flaschen erreicht (1003 $\mathrm{N}$ bei Acryl und $1208 \mathrm{~N}$ bei der Kopfschwarte).

Das Bruchverhalten der Bierflaschen zeigte immer eine Zersplitterung in viele kleine Einzelscherben ( $>30)$, während die oberen ca. $3-6 \mathrm{~cm}$ des in der Hand gehaltenen Flaschenhalses intakt blieben (-Abb. 3). Im Bereich des Etiketts wurden die Glasscherben meist im Verbund zusammengehalten. Der Flaschenboden ist ebenfalls jeweils in

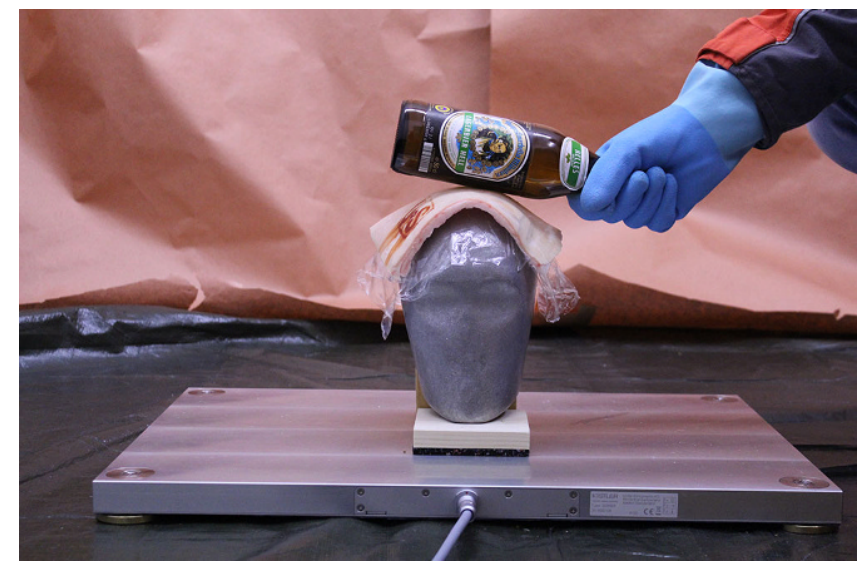

Abb. $2 \triangleleft$ Versuchsaufbau

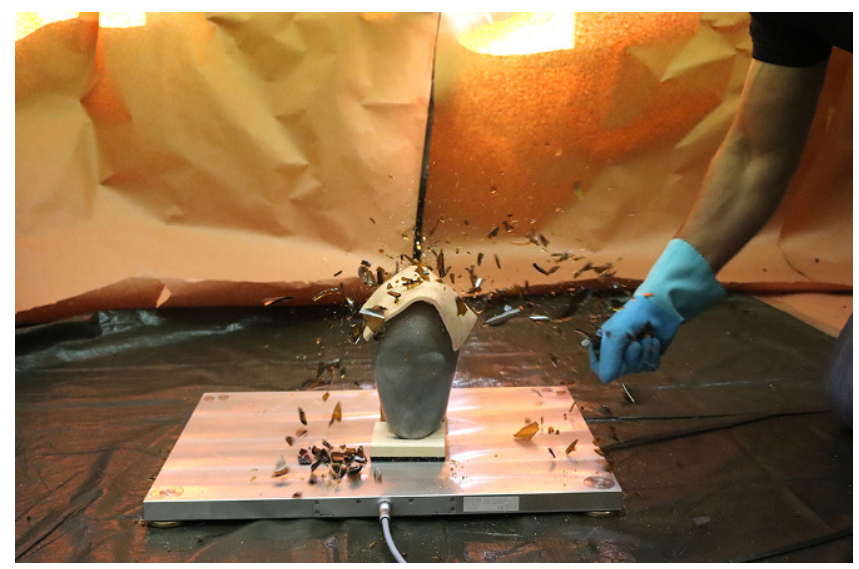

Abb. $3<$ Zersplitterung der Bierflasche beim Auftreffen auf den Schädel

mehrere Fragmente zersprungen. Ein charakteristisches Bruchverhalten im Sinne von nachvollziehbaren Bruchlinien oder Scherben konnte nicht festgestellt werden (• Abb. 4).

Sowohl die Acrylhaut als auch die Schweineschwarte zeigten im Bereich der Kontaktstelle eine leichte Kompression des Gewebes. Scharfe Verletzungen konnten in nur 4 Versuchen auf der Schweineschwarte festgestellt werden. Hier wurden oberflächliche, kleine, bis maximal $0,7 \mathrm{~cm}$ lange und lediglich wenige Millimeter tiefe (ca. $2 \mathrm{~mm}$ ) in das Surrogat eindringende, scharfrandige Verletzungen festgestellt (• Abb.5).

Die typische Kraftübertragung während eines Schlags mit der Bierflasche vollzog sich in einer äußerst kurzen Zeitspanne. Innerhalb von weniger als $4 \mathrm{~ms}$ wurden die durch den Schlag aufgebrachten Kräfte auf den Kopf übertragen. Exemplarisch sind in $\bullet$ Abb. 6 die Kraftverläufe verschiedener Schläge dargestellt.

\section{Diskussion}

Die biomechanisch-forensische Analyse von Verletzungsvorgängen in Form von Schlägen mit einem Gegenstand aus Glas (Flaschen, Trinkgefäße usw.) kann auf verschiedenen physikalischen Parametern basieren und schließt die Bewertung stumpfer sowie evtl. scharfer Gewalt ein. Im Fall einer Stoßeinwirkung mittels eines intensiven Schlags mit einer Bierflasche ist ausschlaggebend, ob beim Stoß zunächst der Schädel oder die Flasche zu Bruch geht - eine Schädelfraktur kann aufgrund der möglichen Komplikationen (insbesondere intrakraniellen Blutungen) zu einer Gefahr für das Leben der betroffenen Person führen, ein Bruch der Flasche verhindert im Endeffekt eine weitere signifikante stumpfe (Stoß-)Einwirkung, und in weiterem Verlauf des Vorgangs steht dann die scharfe Einwirkung im Vordergrund. Um eine objektive Bewertungsgrundlage $\mathrm{zu}$ ermöglichen, wurde in der vorliegenden Studie die Stoßkraft bei Schlägen auf ein Kopf- 
modell gemessen. Das hier zum Einsatz kommende Messverfahren wurde bereits in früheren Untersuchungen zum Thema der potenziellen Verletzungsgefahr bei Schlägen gegen den Kopf von den Autoren angewandt [8, 9]. Grundlage der Bewertung ist die Gegenüberstellung der gemessenen Parameter und der biomechanischen Toleranzgrenzen des jeweiligen Gewebes. Werden Letztere erreicht bzw. überschritten, so kommt es $\mathrm{zu}$ entsprechenden Verletzungen der Haut, Knochen sowie des darunter liegenden Gewebes. Insbesondere auch bei einer hohen Kopfbeschleunigung (mit Interaktion zwischen dem Gehirn und dem Schädel) kann es ferner zu Verletzungen im Bereich des Zentralnervensystems kommen. Bedingt durch individuelle anatomische Unterschiede und verschiedenartige Untersuchungsmethoden werden die Toleranzgrenzen der Haut $[3,6,7,16-18]$ und der Schädelknochen [2, 13, 14, 19] in wissenschaftlichen Veröffentlichungen unterschiedlich angegeben. Hauptgründe für divergierende Resultate sind die experimentell vorbestimmte Art und Weise sowie die Dauer der jeweiligen Belastung, bezogen auf die Kontaktfläche. Unterschiedliche, teils statische und teils dynamische Messmethoden mit differierenden Belastungen ergeben entsprechend variierende Toleranzgrenzen. Auch werden in den publizierten Arbeiten z.T. unterschiedliche physikalische Größen zur Definition der Frakturtoleranzen herangezogen, was eine direkte Vergleichbarkeit zusätzlich erschwert. Im Fall eines Bierflaschenschlags gegen den menschlichen Schädel handelt es sich um eine sehr kurze Kontaktzeit (max. $4 \mathrm{~ms}$ ) sowie eine relativ kleine Kontaktfläche (jeweils konvexe Oberflächen des Schlagobjekts und des Kopfes/Schädels), sodass die in der Literatur angegebenen Parameter nur in Relation zu unseren Versuchen interpretiert werden können. Auch spielt das verwendete organische Material oder Surrogat eine wichtige Rolle und muss bei der rechtsmedizinischen Interpretation publizierter Daten mitberücksichtigt werden.

Die maximal mögliche Kraftentwicklung beim Bierflaschenschlag lag in unseren Versuchen knapp über $1200 \mathrm{~N}$.

Rechtsmedizin 2021 · 31:494-500 https://doi.org/10.1007/s00194-021-00461-0

(c) Der/die Autor(en) 2021

C. Nentwig · A. Schütz · S. Steinhoff · J. Adamec · S. N. Kunz

Forensisch-biomechanische Aspekte von gebrauchten, leeren 0,5I-Bierflaschen aus Glas als Schlagwaffe gegen den Schädel

\section{Zusammenfassung}

Hintergrund. Die rechtsmedizinische Begutachtung von Verletzungen im Kopfbereich durch Schläge mit Bierflaschen aus Glas ist oftmals die Folge einer körperlichen Auseinandersetzung in Schankgaststätten. Im Rahmen der juristischen Aufarbeitung eines entsprechenden Sachverhalts stellen sich die Fragen nach der tatsächlich erfolgten Tathandlung und der akuten sowie potenziellen Lebensgefahr einer getroffenen Person.

Material und Methoden. Hinsichtlich ihres Bruchverhaltens und ihres Verletzungspotentials bei Schlägen auf den Schädel wurden 30 gebrauchte, leere Bierflaschen der Augustiner Brauerei München untersucht. Manuell wurden die Glasflaschen auf einen DummyKopf aus Aluminium geschlagen, welcher mit einem Acryl-Kopfhautsurrogat und alternativ mit Schweineschwarte überzogen und auf einer Kistler-Kraftmessplatte fixiert war. Im Fokus der Untersuchungen standen die maximal übertragbare Kraft auf den Schädel sowie die Bruchschwelle und das Bruchverhalten der Glasflaschen. Ergebnisse. Die auf den Schädel übertragene Kraft steht in Wechselwirkung mit dem
Bruchverhalten des Schlagwerkzeugs und den hierdurch entstehenden Verletzungen. Die Intensität stumpfer Verletzungen steigt mit zunehmender Kontaktkraft bis zum Niveau der Frakturtoleranz der Bierflaschen. Kommt es zu einem Bruch der Flasche, so steigt das Risiko für scharfe Verletzungen bei gleichzeitiger Intensitätsreduktion der Verletzungen durch stumpfe Gewalt. Schläge mit einer gebrauchten 0,5-IBierflasche gegen den Kopf einer Person sind grundsätzlich in der Lage, Frakturen des Gesichtsschädels hervorzurufen. Das Risiko der Entstehung von Frakturen des Hirnschädels ist demgegenüber, zumindest bei Schlägen mit der Seite der Flasche, als äußerst unwahrscheinlich einzuschätzen. Eine abschließende Beurteilung des Gefährdungspotenzials einer konkreten Behandlung kann in jedem Fall erst nach Kenntnis sämtlicher den Einzelfall betreffender Informationen und Variablen erfolgen.

Schlüsselwörter

Rechtsmedizin · Schlag mit Gegenstand . Stumpfe Gewalt - Scharfe Gewalt . Verletzungspotenzial

\section{Forensic and biomechanical aspects of used, empty $0.5 \mathrm{I}$ beer glass bottles as a striking weapon against the skull}

\section{Abstract}

Background. The forensic medical assessment of head injuries from blows with beer bottles is an important aspect in the context of legal prosecution of physical confrontations in bars or restaurants. Especially the forensic assessment of the injury potential of such actions can be essential.

Material and methods. In the study 30 used, empty beer bottles from the Augustiner Brewery in Munich were examined with respect to their injury risks when used as a striking weapon to the head. The glass bottles were manually hit on a dummy skull made of aluminium, which was covered with an acrylic and porcine scalp surrogate. The target was fixed onto a Kistler force measuring plate.

Results. The force transmitted to the skull correlated with the breaking behavior of the striking weapon and the resulting injuries. The intensity of blunt injuries increased with increasing contact force up to the level of fracture tolerance of the beer bottles. If the bottle broke there was a higher risk of sharp injuries but a lower risk of injuries from blunt force trauma. Blows with a used $0.5 \mathrm{I}$ beer bottle can cause fractures of the facial bones. The probability of skull cap fractures appears to be very low. A final assessment regarding the risk of life-threatening injuries can only be made after knowledge of all the information and variables of the case in question.

\section{Keywords}

Forensic medicine $\cdot$ Blows with object . Blunt force trauma - Sharp force trauma. Physical violence 
Tab. 1 Schläge mit der Seite einer 0,5-I-

Bierglasflasche auf Acryl - Aluminium

Acryl-Kopfhautsurrogat

\begin{tabular}{|c|c|c|c|c|c|}
\hline \multicolumn{3}{|c|}{ Acryl-Kopfhautsurrogat } & Nr. & Gebrochen & Kraft in Newton \\
\hline Nr. & Gebrochen & Kraft in Newton & 20.3 & - & 358,15 \\
\hline 1.1 & - & 520,74 & 20.4 & - & 877,44 \\
\hline 1.2 & - & 606,82 & 20.5 & $\mathrm{Ja}$ & 521,23 \\
\hline 1.3 & $\mathrm{Ja}$ & 531,92 & \multirow{4}{*}{\multicolumn{3}{|c|}{$\begin{array}{l}\text { Die fett gedruckten Werte kennzeichnen die } \\
\text { übertragene Kraft bei Zerbrechen der Glasfla- } \\
\text { schen. } \\
{ }^{2} \text { Messfehler }\end{array}$}} \\
\hline 2.1 & - & 916,32 & & & \\
\hline 2.2 & - & 877,52 & & & \\
\hline 2.3 & $\mathrm{Ja}$ & 921,76 & & & \\
\hline
\end{tabular}

$2.3-921,76$
35

3 Ja 368,26

$\begin{array}{lll}4.1 & - & 817,50\end{array}$

$\begin{array}{lll}4.2 & - & 987,42\end{array}$

$4.3 \mathrm{Ja} \quad \mathbf{8 8 3 , 5 3}$

\begin{tabular}{l|l|l}
5.1 & - & 836,84
\end{tabular}

$\begin{array}{lll}5.2 & \mathrm{Ja} & 669,87\end{array}$

$6 \quad \mathrm{Ja} \quad 342,86$

$\begin{array}{lll}7.1 & - & 671,75\end{array}$

$\begin{array}{lll}7.2 & - & 826,30\end{array}$

$\begin{array}{lll}7.3 & - & 766,39\end{array}$

$\begin{array}{lll}7.4 & - & 815,13\end{array}$

$\begin{array}{lll}7.5 & - & 933,96\end{array}$

$\begin{array}{lll}7.6 & - & 1003,59\end{array}$

$\begin{array}{lll}7.7 & - & 954,12\end{array}$

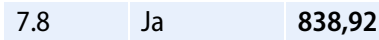

8 Ja $\quad 318,85$

9 Ja $\quad 439,98$

10 Ja 494,90

$11.1 \quad$ - $\quad 607,90$

$11.2 \mathrm{Ja} \quad 460,66$

12.1 - $\quad-^{\mathrm{a}}$

$\begin{array}{lll}12.2 & \mathrm{Ja} & 386,84\end{array}$

13 Ja 369,67

14 Ja 558,25

15 Ja 464,49

16 Ja 518,45

17 Ja $\quad 405,51$

$\begin{array}{lll}18.1 & - & 143,12\end{array}$

$18.2-311,65$

$18.3 \quad-\quad 377,62$

$\begin{array}{lll}18.4 & - & 472,07\end{array}$

18.5 Ja $\quad 518,32$

$\begin{array}{lll}19.1 & - & 778,65\end{array}$

19.2 - $\quad 773,55$

$\begin{array}{lll}19.3 & - & 429,23\end{array}$

$19.4 \quad-\quad 810,18$

$\begin{array}{lll}19.5 & - & 779,63\end{array}$

$19.6 \quad-\quad 812,20$

$\begin{array}{lll}19.7 & - & 860,72\end{array}$

19.8 Ja $\quad 632,92$

$20.1 \quad-\quad 598,41$

$20.2 \quad-\quad 813,82$
Tab. 1 (Fortsetzung)

Acryl-Kopfhautsurrogat

Bei experimentell ermittelten Frakturtoleranzen des Stirnbeins von $4,0 \mathrm{kN}$ [14], des Hinterhauptbeins von $13,6 \mathrm{kN}$ [19], des Scheitelbeins von 5,8-17,0 kN [2] und des Schläfenbeins von $6,1 \mathrm{kN}$ [13] ist es äußerst unwahrscheinlich, durch den Schlag mit einer 0,5-1-Bierflasche eine Fraktur des Hirnschädels zu bewirken.

Bezogen auf die Gesichtsknochen kann die strukturelle Festigkeit der Flaschen die der menschlichen Knochen überschreiten. 6 unserer insgesamt 89 Schlagversuche erreichten einen Wert über $1000 \mathrm{~N}$, was die Bruchkraft des Jochbeins überschreitet [14] und zu entsprechenden Frakturen führen kann. Das Nasenbein hat eine Frakturschwelle $<0,5 \mathrm{kN}$ [2] und ist somit durch eine Bierflasche leicht zu frakturieren. Diese Ergebnisse decken sich mit epidemiologischen Studien [5] und Falldarstellungen [11, 12], wobei die Intensität und Häufigkeit von Hautverletzungen in diesen Publikationen stärker repräsentiert waren als bei unseren Versuchen. In einem Teil dieser Fälle wurden eine 0,75-1-und eine 1,0-1-Glasflasche als Schlagwerkzeuge verwendet. Aufgrund des höheren Gewichtes ist hier mit einer höheren Kraftentwicklung und -übertragung mit entsprechend abweichendem Verletzungspotenzial zu rechnen [15]. In einer Arbeit von Bollinger et al. [4] wird Glasflaschen bei anderem Versuchsaufbau das Potenzial zugesprochen, Schädelkalottenverletzungen hervorzurufen. Auch wenn Frakturen des Schädelknochens nicht ausgeschlossen werden können, so zeigen unsere Ergebnisse, dass die Wahrscheinlichkeit eines solchen Ereignisses zumindest in der von uns untersuchten Situation mit Einwirkung der Seitenfläche der Flasche äußerst ge-
Tab. 2 Schläge mit der Seite einer 0,5-I-

Bierglasflasche aufSchweineschwarte -Aluminium

\begin{tabular}{|c|c|c|}
\hline \multicolumn{2}{|c|}{ Schweineschwarte } & \multirow[b]{2}{*}{777,20} \\
\hline 21 & - & \\
\hline 21.2 & - & 832,98 \\
\hline 21.3 & - & 850,69 \\
\hline 21.4 & - & 912,87 \\
\hline 21.5 & - & 1025,44 \\
\hline 21.6 & - & 900,11 \\
\hline 21.7 & - & 1057,09 \\
\hline 21.8 & Ja & 951,87 \\
\hline 22 & Ja & 394,02 \\
\hline 23.1 & - & 586,57 \\
\hline 23.2 & - & 868,22 \\
\hline 23.3 & - & 1131,83 \\
\hline 23.4 & $\mathrm{Ja}$ & 885,70 \\
\hline 24.1 & - & 835,39 \\
\hline 24.2 & - & 863,84 \\
\hline 24.3 & - & 994,67 \\
\hline 24.4 & Ja & 827,58 \\
\hline 25 & Ja & 845,24 \\
\hline 26 & - & 622,17 \\
\hline 26.2 & - & 837,01 \\
\hline 26.3 & Ja & 478,15 \\
\hline 2.71 & - & 544,72 \\
\hline 27.2 & - & 580,88 \\
\hline 27.3 & - & 528,37 \\
\hline 27.4 & - & 881,06 \\
\hline 27.5 & - & 1001,06 \\
\hline 27.6 & - & 1208,60 \\
\hline 27.7 & - & 943,91 \\
\hline 27.8 & Ja & 850,76 \\
\hline 28 & Ja & 795,80 \\
\hline 29.1 & - & 895,09 \\
\hline 29.2 & - & 498,81 \\
\hline 29.3 & - & 936,39 \\
\hline 29.4 & - & 599,26 \\
\hline 29.5 & - & 892,51 \\
\hline 29.6 & - & 1168,50 \\
\hline 29.7 & - & 763,47 \\
\hline 29.8 & $\mathrm{Ja}$ & 903,10 \\
\hline 30 & Ja & 576,12 \\
\hline
\end{tabular}

Mittelwert der Bruchkraft nach Versuchen 01-10: $5810,85 \mathrm{~N}: 10=581,085 \mathrm{~N}$

Mittelwert der Bruchkraft nach Versuchen

$11-20: 4836,34 \mathrm{~N}: 10=483,63 \mathrm{~N}$

Mittelwert der Bruchkraft nach 20 Versuchen

532,36 N $(10.647,19 \mathrm{~N}: 20=532,36 \mathrm{~N})$

Mittelwert der Bruchkraft nach Versuchen 21-30: 7508,33 N: 10 = 750,83 N $\rightarrow$ Schwei-

neschwarte

Mittelwert der Bruchkraft nach 30 Versuchen: $\mathbf{6 0 5}, 18 \mathrm{~N}(18.155,52 \mathrm{~N}: 30=605,18 \mathrm{~N})$ 


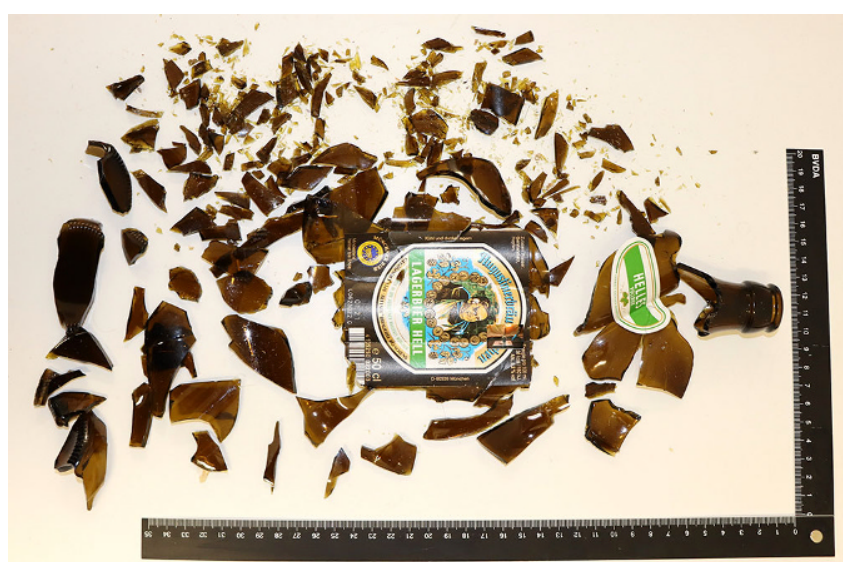

Abb. 4 ム Resultierende Frakturstücke nach Bierflaschenschlag mit zylindrischer Seitenfläche

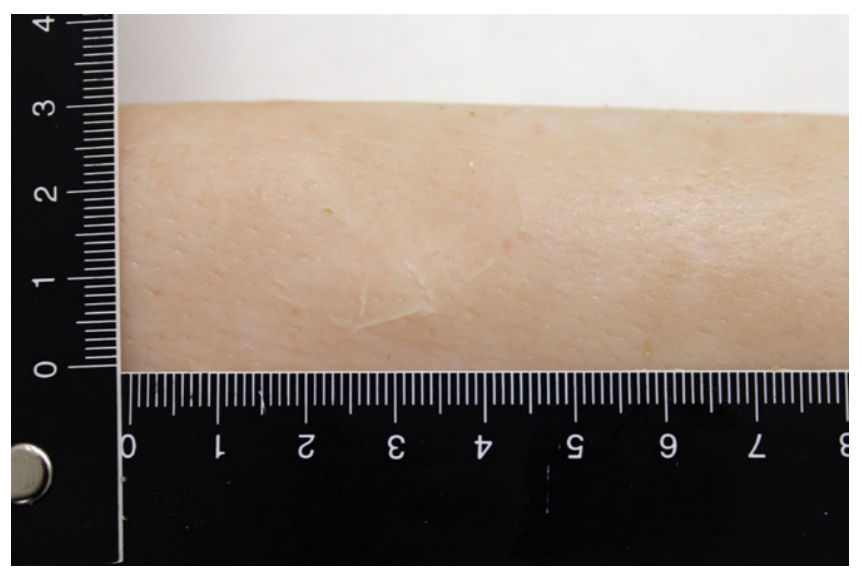

Abb. $5 \Delta$ Defekte an der Schweineschwarte nach Bierflaschenschlag

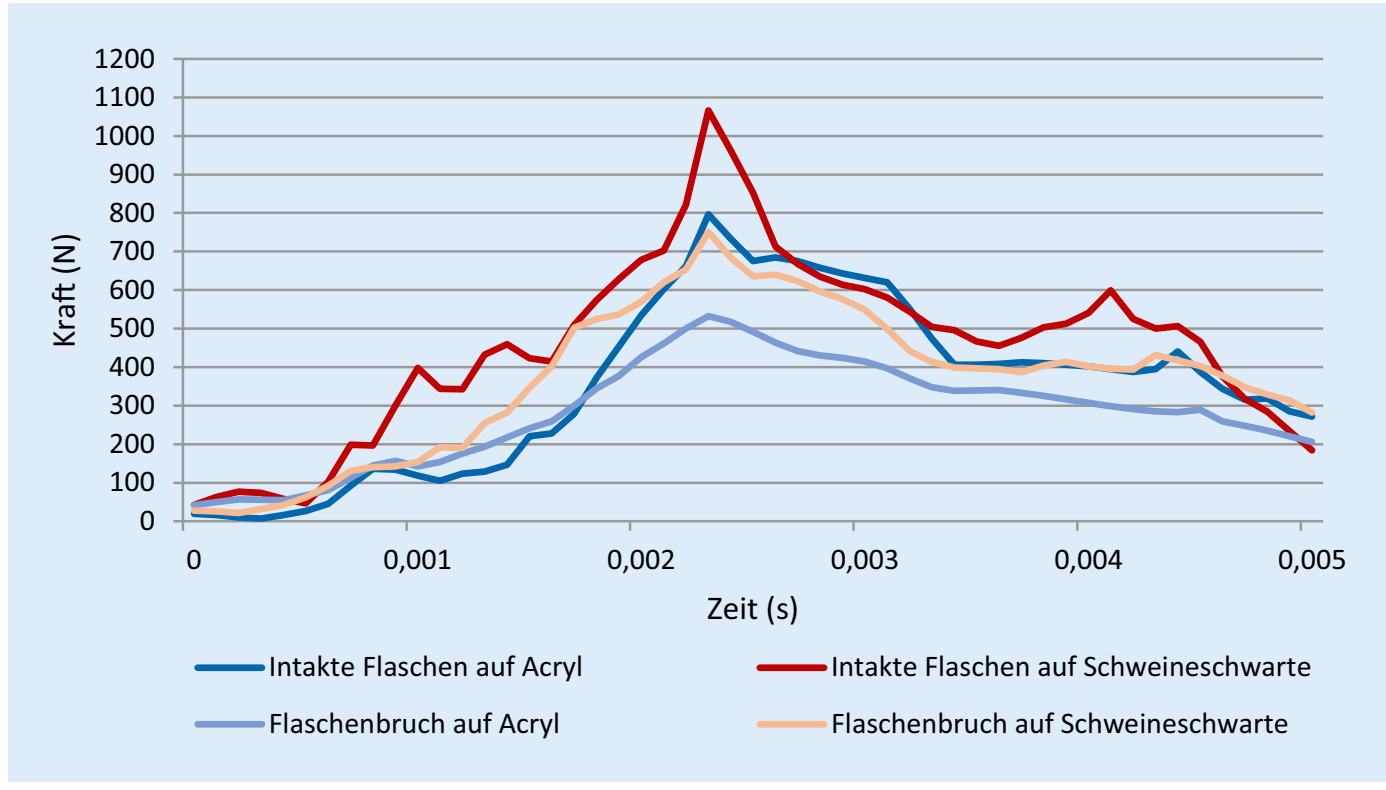

Abb. $6 \triangleleft$ Kraftverlaufskurven einer Schlagbewegung mit einer 0,5-I-Bierglasflasche gegen den Schädel ring ist. Dies steht mit Falldarstellungen und Ergebnissen von PMHS-Versuchen (PMHS: „postmortal human subjects") von Madea et al. [12, 13] in Einklang und sollte bei der Beurteilung der potenziellen Lebensgefahr eines Schlages mit einer Bierglasflasche berücksichtigt werden, zusammen mit der Tatsache, dass tödliche Verletzungen infolge von Schlägen mit Glasflaschen selten sind eine Einschätzung von Madea [10], die sich mit unserer Erfahrung deckt.

Kommt es bei Kontakt des Schlagwerkzeugs mit dem Schädel zu einem Zerbrechen der Bierflasche, so geht durch den Bruchvorgang ein Teil der übertragenen Energie verloren. Das bedeutet, dass eine intensiv durchgeführte
Schlagbewegung mit Zerbrechen der Flasche beim Aufprall auf dem Kopf $\mathrm{zu}$ einer wesentlich geringeren Kraftübertragung und somit zu einer relevant geringeren Verletzungsgefahr bezüglich stumpfer Gewalt führt als ein Schlag, der mit einer Intensität knapp unterhalb des Bruchniveaus der Flasche durchgeführt wird. Gleichzeitig steigt jedoch die Wahrscheinlichkeit von Verletzungen durch scharfe Gewalt, was jedoch nach derzeitigem Kenntnisstand in Bezug auf die potenzielle Lebensgefahr eine untergeordnete Rolle zu spielen scheint. Bezogen auf Verletzungen durch scharfe Gewalt entstehen diese beim Zerbrechen der Flasche durch die Druck-SchnittKombination und durch beschleunigte
Glassplitter mit noch ausreichender Restenergie (d.h. ein ausreichendes Gewicht bei entsprechender Beschleunigung). Anhand der vorliegenden Ergebnisse sind oberflächliche Verletzungen durch entstandene Glasfragmente zwar möglich, tiefgreifende Schnittverletzungen konnten bei senkrechter Schlagbewegung im Rahmen unserer Versuche jedoch nicht beobachtet werden.

In den Experimenten wurden die Schläge mit leeren Bierflaschen durchgeführt, da hier eine höhere maximale Kraftübertragung zu erwarten ist. Gefüllte Bierflaschen haben eine niedrigere Frakturtoleranzschwelle, brechen bei geringerer Krafteinwirkung und haben somit ein insgesamt etwas geringeres 
Verletzungspotenzial [4]. Bei der Beurteilung der potenziellen Lebensgefahr einer Verletzung mit Bierflaschen sollte daher auch immer an eine leere Flasche gedacht werden.

\section{Einschränkungen}

In der vorliegenden Arbeit wurde eine relativ geringe Anzahl von Versuchen durchgeführt. Allerdings ist es bekannt, dass ballistische Testreihen auf physikalischen Grundlagen beruhen und somit auch eine hohe Fallzahl eine nur geringe Abweichung der Grundparameter wie Masse, Geschwindigkeit oder Bruchverhalten zur Folge hat. Für die rechtsmedizinische Beurteilung und Interpretation dieser speziellen Art der Gewalteinwirkung würde eine geringgradige $\mathrm{Ab}$ weichung vom Ergebnis keine relevanten Auswirkungen haben.

Die manuelle Durchführung der Versuche erlaubt realitätsnahe Ergebnisse und ermöglicht so eine praxisnahe forensisch-biomechanische Interpretation der erworbenen Daten.

Die Schläge wurden in der vorliegenden Versuchsreihe gegen einen unzerbrechlichen Kopf-Dummy geführt, was keine Beobachtung von Frakturen ermöglicht. Die diskutierten Schlussfolgerungen bezüglich des Verletzungspotenzials wurden durch einen Literaturvergleich gezogen.

Im Gegensatz $\mathrm{zu}$ einem menschlichen Schädel, der innerhalb seiner Belastungsgrenzen auch zumindest geringfügig elastisch verformbar ist, ist der Aluminiumkern des Dummy-Kopfmodels starr. Dies begünstigt eine kurze Stoßdauer und eine geringe Kontaktfläche (d.h. bei gegebener Kraft eine hohe Spannung = Kraft pro Fläche). Bei stark konvexer Oberfläche einer Bierflasche dürfte jedoch die Präsenz eines verformbaren Hautsurrogats ausschlaggebend sein, durch eine Verformung der Kalotte von insgesamt wenigen Millimetern (wobei auf die lokale Verformung mit Änderung der Kontaktfläche nur ein Bruchteil entfallen dürfte) wie experimentell festgestellt [19], ist eine große Veränderung des Verhältnisses zwischen Kraft und Kontaktfläche oder der Stoßdauer nicht zu erwarten. Da- für, dass die verwendete Messmethode nicht zu einer Unterschätzung des Verletzungspotenzials führt, zeigt unsere Auswertung konkreter Fälle von Maßkrugschlägen [1]. Hier wurden weniger schwere Verletzungen festgestellt, als die Messresultate erwarten ließen, die mit einem vergleichbaren Set-up gewonnen wurden.

\section{Fazit}

Schläge mit einer leeren 0,5-1-Bierflasche aus Glas gegen den menschlichen Schädel können zu stumpfen und scharfen Verletzungen führen.

Bei Kontakt der Glasflasche im Gesichtsbereich (v. a. Nasenbein, Jochbein) sind Knochenverletzungen durchaus möglich, bei Aufschlagen am Hirnschädel sind Frakturen als äußerst unwahrscheinlich einzustufen.

Bricht die Bierflasche beim Auftreffen auf den Schädel, so führt dies zu einer deutlichen Reduktion der Kraftübertragung mit Risikominderung stumpfer Verletzungen. Gleichzeitig erhöht sich durch die beschleunigten Scherben die Wahrscheinlichkeit scharfer Verletzungen.

Die rechtsmedizinische Beurteilung körperlicher Auseinandersetzungen geht in der Regel mit einer hohen Anzahl unbekannter Variablen einher. Im Fall eines Bierflaschenschlags gegen den Kopf sind dies meist der Gebrauchszustand der Flasche, die individuelle Dicke des Knochenschädels, die tatsächliche Kontaktfläche und die Schlaggeschwindigkeit. Daher ist es ratsam, eine forensischbiomechanische Beurteilung von Verletzungen durch Bierflaschen erst nach Kenntnis sämtlicher Informationen den Hergang betreffend durchzuführen.

\section{Korrespondenzadresse}

\section{Prof. Dr. S. N. Kunz}

Institut für Rechtsmedizin des Universitätsklinikums UIm

Prittwitzstr. 6, 89075 Ulm, Deutschland

Sebastian.Kunz@uniklinik-ulm.de

Funding. Open Access funding enabled and organized by Projekt DEAL.

\section{Einhaltung ethischer Richtlinien}

Interessenkonflikt. C. Nentwig, A. Schütz, S. Steinhoff, J. Adamec und S.N. Kunz geben an, dass kein Interessenkonflikt besteht.

Für diesen Beitrag wurden von den Autoren keine Studien an Menschen oder Tieren durchgeführt. Für die aufgeführten Studien gelten die jeweils dort angegebenen ethischen Richtlinien.

Open Access. Dieser Artikel wird unter der Creative Commons Namensnennung 4.0 International Lizenz veröffentlicht, welche die Nutzung, Vervielfältigung, Bearbeitung, Verbreitung und Wiedergabe in jeglichem Medium und Format erlaubt, sofern Sie den/die ursprünglichen Autor(en) und die Quelle ordnungsgemäß nennen, einen Link zur Creative Commons Lizenz beifügen und angeben, ob Änderungen vorgenommen wurden.

Die in diesem Artikel enthaltenen Bilder und sonstiges Drittmaterial unterliegen ebenfalls der genannten Creative Commons Lizenz, sofern sich aus der Abbildungslegende nichts anderes ergibt. Sofern das betreffende Material nicht unter der genannten Creative Commons Lizenz steht und die betreffende Handlung nicht nach gesetzlichen Vorschriften erlaubt ist, ist für die oben aufgeführten Weiterverwendungen des Materials die Einwilligung des jeweiligen Rechteinhabers einzuholen.

Weitere Details zur Lizenz entnehmen Sie bitte der Lizenzinformation auf http://creativecommons.org/ licenses/by/4.0/deed.de.

\section{Literatur}

1. Adamec J, Dorfner P, Graw M, Lochner S, Kunz SN (2020) Injury potential of one-litre beer steins. Int J Legal Med 133(4):1075-1081

2. Allsop D, Perl R, Warner C (1991) Force/deflection and fracture characteristics oft he Temporoparietal region oft he human head. SAE Technical Paper. 912907

3. Bader DL, Bowker P (1983) Mechanical characteristics of skin and underlying tissues in vivo. Biomaterials 4:305-308

4. Bollinger SA, Ross S, Oesterhelweg L, Thali MJ, KneubuehI BP (2009) Are full or empty beer bottles sturdier and does their fracture-threshold suffice to break the human skull? J Forensic Leg Med 16:138-142

5. Coomaraswamy KS, Sheperd JP (2003) Predictors and severity of injury in assaults with barglasses and bottles. Inj Prev 9:81-84

6. Edwards C, Marks R (1995) Evaluation of biomechanical properties of human skin. Clin Dermatol 13:375-380

7. Holzmann H, Korting GW, Kobelt D, Vogel HG (1971) Prüfung der mechanischen Eigenschaften von menschlicher Haut in Abhängigkeit von Alter und Geschlecht. Arch Klein Exp Derm 239:355-367

8. Kunz SN, Graw N, Adamec J (2020) Schädelverletzungen durch 1 l Bierkrüge aus Ton. Forensische und biomechanische Aspekte. Rechtsmedizin 30:137-143

9. Kunz SN, Tutsch-Bauer E, Graw M, Adamec J (2016) Biomechanische Aspekte des 0.5I Tonkrugs als Schlagwaffe. Rechtsmedizin 26:189-196 
10. Madea B, Lignitz E (2009) A response to "S.A. Bollinger, S. Ross, L. Osterhelweg, M.J. Thali, B.P. Kneubuehl, Are full or empty beer bottles sturdier and does their fracture-threshold suffice to break the human skull?". J Forensic Leg Med. 16 (2009) 138-142. J Forensic Leg Med 16:432

11. Madea B, Lignitz E, Weinke H (1993) Schädelverletzungen durch Schlag mit Glasflaschen. Arch Kriminol 192:73-79

12. Madea B, Schmidt PH, Lignitz E, Padosch SA (2005) Skull injuries caused by blows with glass bottles. Forensic Pathol Rev 2:29-41

13. Montava M, Masson C, Lavieeille JP, Mancini J, Soussan J,ChaumoitreK, Arnoux P(2016)Temporal bone fracture under lateral impact: biomechanical and macroscopicevaluation. Med Biol Eng Comput 54:351-360

14. Nahum AM, Gatts CW, Danforth JP (1968) Impact tolerance oft he skull. Proc, 12th Stapp Car Crash Conference, S302-317

15. Sprenger FD, Siegenthaler L, Kneubuehl BP, Jackowski C (2016) The influence of striking object characteristics on the impact energy. Int J Legal Med 130:835-844

16. Sugihara T,OhuraT,HommaK, Igawa HH(1991)The extensibility in human skin: variation according to age and site. Br JPlast Surg 44:418-422

17. Vogel HG (1987) Age dependence of mechanical and biomechanical properties of human skin. Part I: Stress-strain experiments, skin thickness and biomechanical analysis. Bioeng Skin 3:67-91

18. Vogel HG (1987) Age dependence of mechanical and biochemical properties of human skin. Part II: Hysteresis, relaxation, creep and repeat strain experiments. Bioeng Skin 3:141-176

19. Yoganandan N, Pintar A, Sances JR, Walsh PR,

Ewing C, Thomas D, Snyder RG (1995) Biomechanics of skull fracture. J Neurotrauma 12:659-668

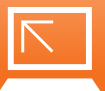

\section{Alle Inhalte von springermedizin.de online im Volltext lesen}

Mit e.Med Interdisziplinär - dem Kombi-Abo von SpringerMedizin.de - können Sie jederzeit auf alle Inhalte zugreifen, die Sie für Ihren Praxis- oder Klinikalltag benötigen. Sie sind immer aktuell informiert - über die neuesten Publikationen, wichtige Studien oder innovative Therapieverfahren.

Die Vorteile von e.Med Interdisziplinär:

- Gestalten Sie Ihre Fortbildung nach Ihren Bedürfnissen:

e.Med Interdisziplinär bietet Ihnen Online-Zugang zu 600 CME-Fortbildungskursen aller Fachrichtungen

- Sie entscheiden, was Sie lesen möchten:

Alle Fachzeitschriften stehen digital im Volltext zur Verfügung.

- Finden Sie die gewünschten Informationen auch in englischen Publikationen Sie können in mehr als 500 englischsprachigen Fachzeitschriften online recherchieren und auf die Suchergebnisse uneingeschränkt im Volltext zugreifen. - Auf Wunsch erhalten sie darüber hinaus eine gedruckte Fachzeitschrift nach Wahl.

NEU: Mit den fachspezifischen e.Med-Abos können Sie sich effizient innerhalb Ihres Fachgebietes auf dem Laufenden halten. Das Angebot reicht von AINS bis Radiologie - Sie haben die Wahl!

Testen Sie e.Med kostenlos und unverbindlich!

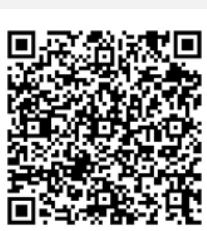

Jetzt informieren unter www.springermedizin.de $\Rightarrow$ „Abos" oder telefonisch unter 0800-77 80777

(Montag bis Freitag, 10 bis 17 Uhr) 